\title{
Functional and gene expression analysis of hTERT overexpressed endothelial cells
}

\author{
Haruna Takano' \\ Satoshi Murasawa ${ }^{1,2}$ \\ Takayuki Asahara 1,2,3 \\ 'Institute of Biomedical Research \\ and Innovation, Kobe, Japan; ${ }^{2}$ RIKEN \\ Center for Developmental Biology, \\ Kobe 650-0047, Japan; ${ }^{3}$ Tokai \\ University of School of Medicine, \\ Tokai, Japan
}

\begin{abstract}
Telomerase dysfunction contributes to cellular senescence. Recent advances indicate the importance of senescence in maintaining vascular cell function in vitro. Human telomerase reverse transcriptase (hTERT) overexpression is thought to lead to resistance to apoptosis and oxidative stress. However, the mechanism in endothelial lineage cells is unclear. We tried to generate an immortal endothelial cell line from human umbilical vein endothelial cells using a no-virus system and examine the functional mechanisms of hTERT overexpressed endothelial cell senescence in vitro. High levels of hTERT genes and endothelial cell-specific markers were expressed during long-term culture. Also, angiogenic responses were observed in hTERT overexpressed endothelial cell. These cells showed a delay in senescence and appeared more resistant to stressed conditions. PI3K/Akt-related gene levels were enhanced in hTERT overexpressed endothelial cells. An up-regulated PI3K/Akt pathway caused by hTERT overexpression might contribute to anti-apoptosis and survival effects in endothelial lineage cells.
\end{abstract}

Keywords: endothelial, telomerase, senescence, oxidative stress, anti-apoptosis, PI3K/Akt pathway

In vitro, normal somatic cells undergo a non-dividing state termed cellular replicative senescence (Harley et al 1990; Hastie et al 1990; Wright and Shay 1992). The erosion of telomeres, a reverse transcriptase synthesize telomeric DNA, has been suggested to contribute to cellular replicative senescence (Greider 1990; Nakamura et al 1997). It is necessary to increase the number of human endothelial progenitor cells (EPCs) in vitro to obtain large numbers for research and cell transplantation. A potential obstacle to this increase, however, is the fact that EPCs, as well as other somatic cells, have a limited replication lifespan.

Human telomerase reverse transcriptase (hTERT)-transduced differentiated endothelial cell (ECs) exhibit neither evidence of malignant transformation nor loss of functional and morphologic characteristics of parental cells (Yang et al 1999). These findings support the stability of endothelial lineage cells after hTERT overexpression. We previously reported on the therapeutic potential of adenoviral hTERT overexpressed endothelial lineage cells in an ischemic hindlimb mouse model (Murasawa et al 2002). However, ectopic hTERT expression by adenovirus transduction was limited to 4 weeks and did not lead to immortal EPCs. We had expected that the enhanced regenerative activity of EPCs by hTERT transduction would provide a novel therapeutic strategy for potential neovascularization in patients with severe ischemic disease. However, the mechanism by which hTERT-transduced EC lines appear more resistant to programmed cell-death and exhibit a survival advantage beyond replicative senescence is elusive. Thus, further investigation of the possible mechanistic pathways is needed.

Here we perform gene transfer with a no-virus system to transduce hTERT genes into human umbilical vein endothelial cells (HUVECs), one of the endothelial lineage 
cell models, to investigate the relation between senescence and telomerase in vitro, determine the possible mechanistic pathways, and investigate the characteristics of these cells for future clinical application.

\section{Materials and methods}

\section{Reagents}

Recombinant human $\mathrm{VEGF}_{165}$ and bFGF were purchased from R\&D Systems Inc. (Minneapolis, MN, USA). Hoechst 33342 solution was purchased from Dojin Chemical Laboratory (Kumamoto, Japan).

\section{Cell culture and gene transfer}

HUVECs were grown in endothelial cell growth media EGM-2 Bullet kit (CAMBREX, Walkersville, MD, USA) supplemented with $2 \%$ fetal bovine serum (CAMBREX). The cDNA encoding hTERT was cloned downstream of the human cytomegalovirus (CMV) promoter in pcDNA3.1(+) (Invitrogen, Carlsbad, CA, USA) with Lipofectamine ${ }^{\mathrm{TM}}$ 2000 (Invitrogen). Forty-eight hours after transfection, the transfected-cell populations were selected with $600 \mu \mathrm{g} / \mathrm{mL}$ of G418 (GIBCO, Grand Island, NY). Cells transfected with empty vectors were used as controls. Population doublings (PD) were calculated as follows: $\mathrm{PD}=\log$ (number of cells obtained/initial number of cells)/log 2 .

\section{RT-PCR for telomerase transcripts}

Total RNA was isolated with an RNA extraction kit (Ambion, Austin, TX, USA). DNase digestion was performed after RNA extraction. cDNA was synthesized by AMV Firststrand Synthesis System (Takara Bio, Shiga, Japan). PCR reaction of hTERT and endothelial markers were performed by a system according to the manufacture (BD Biosciences, San Diego, CA and Applied Biosystems, Foster City, CA, USA). The primers for RT-PCR are shown in Table 1.

\section{Senescence-associated (SA)- Galactosidase (Gal) Staining}

$4 \times 10^{4}$ cells were plated on 12-well plates pre-coated with fibronectin and cultivated in growth medium for 24 hours.
Senescence was investigated by a SA-Gal activity assay system according to the manufacture (CALBIOCHEM, San Diego, CA, USA). Quantification of SA-Gal-positive cells was obtained by counting five random fields per dish and assessing the percentage.

\section{Proliferation assay}

$3.5 \times 10^{3}$ cells grown in 96-well plates pre-coated with fibronectin were cultivated in growth medium for 24 hours then in basal medium for another 24 hours. Then the culture medium was changed to basal medium in the presence of angiogenic cytokines for 24 hours. Proliferative activity was evaluated using the MTS assay (Promega, Madison, WI, USA).

\section{Apoptosis assay}

$1 \times 10^{5}$ cells grown in 12 -well plates pre-coated with fibronectin were cultivated in growth medium for 24 hours then in basal medium for another 24 hours. To detect the frequency of cellular apoptosis, fluorescence-labeled Annexin-V-FLUOS staining of transduced HUVECs was performed according to manufacture's instructions (Roche Molecular Biochemicals, Mannheim, Germany). Quantification of Annexin-V positive cells was obtained by counting 5 random fields per dish and assessing the percentage.

\section{Oxidative stress detection assay}

$1 \times 10^{5}$ cells were grown in 12 -well plates pre-coated with fibronectin cultivated in growth medium. After 24 hours' incubation, the cells were incubated with $1 \mathrm{mM} \mathrm{H}_{2} \mathrm{O}_{2}$ in HEPES buffer for 1 hour. Then the medium was changed to growth medium for another 3 hours. Detection of oxidative damage in live cells was performed using the Image-i $\mathrm{T}^{\mathrm{TM}}$ LIVE Green Reactive Oxygen Species (ROS) Detection Kit (Molecular Probes, Eugene, OR, USA). Quantification of ROS detected cells was obtained by counting 5 random fields per dish and assessing the percentage.

\section{Gene array analysis}

Targeted cDNA arrays designed to analyze endothelial cell biology, angiogenesis, apoptosis, cell cycle, and PI3K/Akt pathway (GEArray HS-036, HS-009, HS-603, and HS-058)

Table I List of primer sequences for PCR

\begin{tabular}{lll}
\hline Molecule & Sense (5'-3') & Antiense (5'-3') \\
\hline hTERT & CACCTCACCTCACCCACGCGAAA & CCAAAGAGTTTGCGACGCATGTT \\
hCD3I & AGGACATCCATGTTCCGAGA & TGAACCGTGTCTTCAGGTTG \\
hKDR & CCCTGCCGTGTTGAAGAGTT & GGACAGGGGGAAGAACAAAA \\
heNOS & ATACCATGGCAACCAACGTC & AAAAGCTCTGGGTGCGTATG \\
hGAPDH & GCCCCAGCAAGAGCACAAGA & TAGGCCCCTCCCCTCTTCAA \\
\hline
\end{tabular}


were purchased from SuperArray Bioscience Corporation (Frederick, MD, USA). Hybridization was quantified using a Luminescent Image Analyzer LAS-3000 (Fujifilm, Tokyo, Japan) and data analysis was performed using Science Lab 2001 Image Gauge (Fujifilm) software. The background was subtracted from each representing dot signal, and values were normalized according to the GAPDH signal.

\section{Statistical analysis}

All values are expressed as mean \pm SD. Comparisons of results between different groups were performed using Student's t test.

\section{Results}

\section{hTERT transduced HUVECs expressed} telomerase gene during long term culture hTERT transduced HUVECs (Td/TERTs) were isolated from several donors cell sources. RT-PCR analysis showed that telomerase gene expression was maintained during long term culture, and the level achieved in Td/TERTs was comparable to that expressed by the 293 human embryonic kidney tumor cell lines (Figure 1A). By contrast, mock transduced HUVECs (Td/mocks) did not express endogenous

A

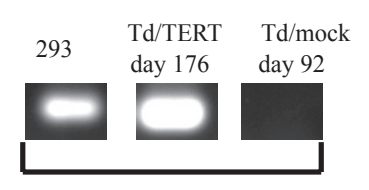

hTERT

$\mathrm{B}$

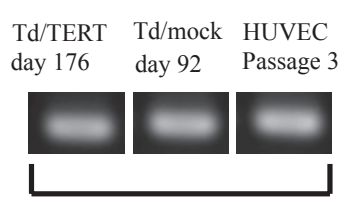

CD31

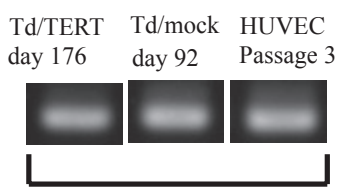

KDR

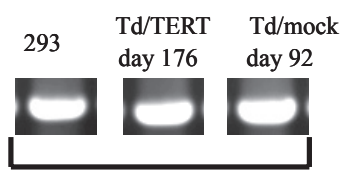

GAPDH

Td/TERT Td/mock HUVEC day 176 day 92 Passage 3

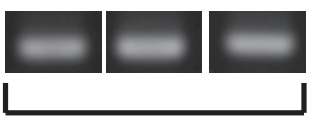

eNOS

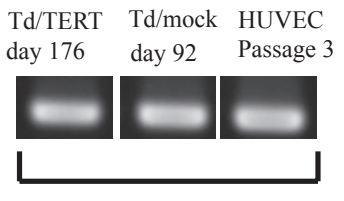

GAPDH
Figure I hTERT transduced HUVECs expressed telomerase genes during long-term culture. RNA samples from transduced HUVECs were analyzed by RT-PCR for gene expression. (A) Expression of hTERT gene in 293 cells served as the positive standard. (B) Expression of endothelial markers. telomerase at early cultures (data not shown). Further, the transduced HUVECs expressed endothelial markers such as CD31, KDR, and eNOS during long term culture (Figure 1B). Sequences of PCR primers are shown in Table 1.

\section{Transduced HUVEC lines maintained endothelial characteristics}

Phenotypes of these cell lines displayed serum-dependence for growth (Figure 2A) and normal contact inhibition

$\mathbf{A}$

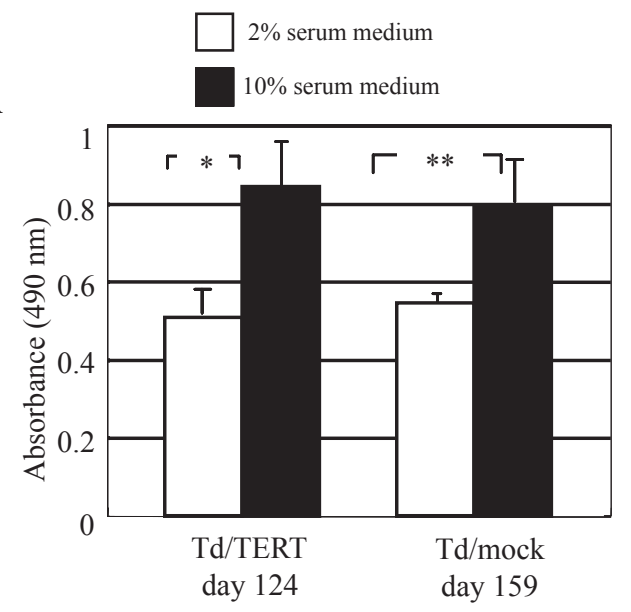

B

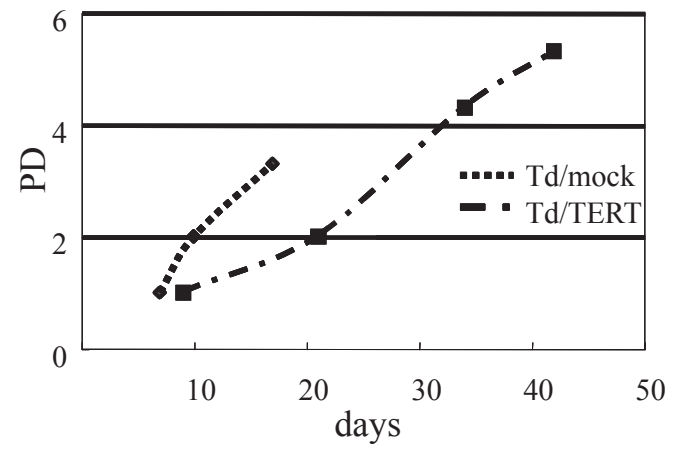

C Td/TERT day 96 Td/mock day 25
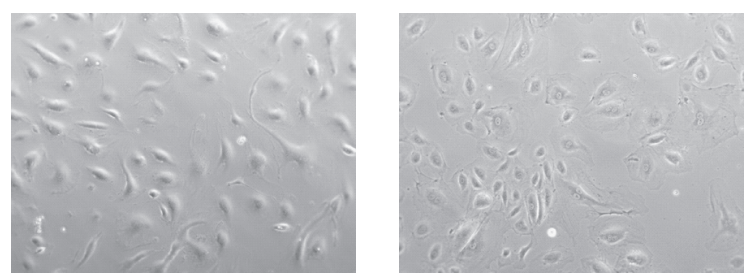

Figure 2 Transduced HUVEC lines maintained endothelial characteristics. (A) Growth of transduced HUVECs, Td/mock (day I59) and Td/TERT (day I24), was inhibited in low serum (*p $<0.001$, ${ }^{* *} \mathrm{p}<0.05$ ). (B) Growth curves of transduced HUVEC.About in a month, most transduced HUVECs showed almost a typical senescent endothelial morphology and stopped continuous growth. However, hTERT overexpression allowed the maintanance of a relatively normal endothelial morphology during the aging process. (C) Phase microscopy demonstrated endothelial morphology of Td/TERTs (day 96) and $\mathrm{Td} /$ mocks (day 25). Original magnification $\times 100$. 
(data not shown). Thus, the transduced HUVEC lines showed no signs of tumorigenic transformation. About in a month, transduced HUVECs ordinary entered the growtharrested state (Figure 2B), and these phenotypes developed an enlarged and flattened morphology (Figure 2C, Td/mocks [day 25]). In contrast, the Td/TERTs (day 96) maintained a relatively normal endothelial morphology during the aging process (Figure 2C). However, some Td/mocks survived more than a month and maintained the same normal endothelial morphology as the Td/TERTs (data not shown).

\section{Transduced HUVEC senescence evaluated by SA-Gal staining}

The cytochemical senescent phenotype was also examined using SA-Gal activity. As expected, hTERT expression facilitated the delay in senescence (Figure 3A, B). During the aging process, transduced HUVECs revealed SA-Gal activity. However, the ratio to total cells in Td/mocks (day 32) was significantly increased compared with that in Td/TERTs (day 105) (41\% $\pm 13 \%$ vs $7 \% \pm 4 \%, p \leq 0.001)$. These results indicated that hTERT overexpression delayed the cells from entering their senescent state.

\section{hTERT overexpression exceeded mitogenic potential.}

Endothelial cell lines have a mitogenic response to angiogenic cytokines. To determine whether Td/TERTs produced angiogenic responses on stimulation by growth factors, we performed angiogenic proliferation assays by treating transduced HUVECs with different cytokines in $\mathrm{VEGF}_{165}$ and bFGF while applying serum-depleted basal EBM-2 medium. As shown in Figure 3C, D, when treated with VEGF $_{165}$ (Figure 3C) and bFGF (Figure 3D), MTS assay showed significantly increased proliferation in Td/TERTs
A
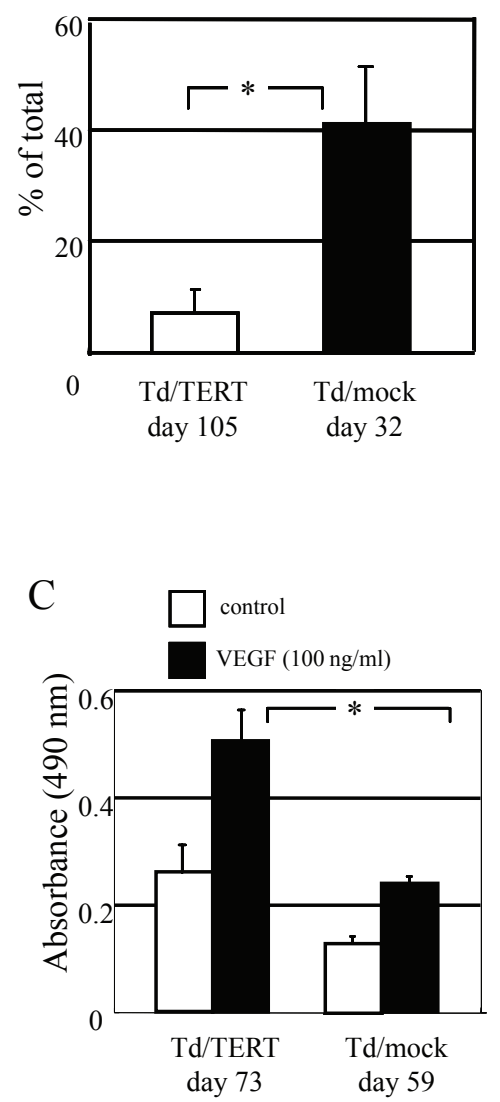

$\mathrm{B}$

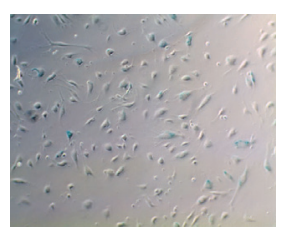

Td/TERT

day 105

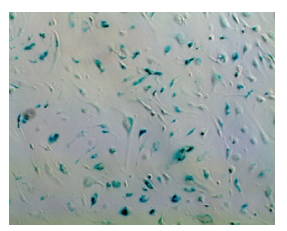

$\mathrm{Td} / \mathrm{mock}$

day 32

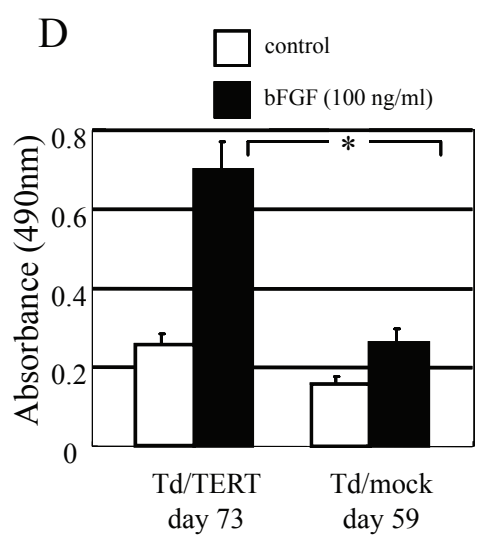

Figure 3 Senescence in transduced HUVECs was evaluated by SA-Gal staining. (A) Quantification of SA-Gal-positive cells in transduced HUVECs obtained by counting 5 random fields per dish ( $p<0.00$ I vs Td/mocks). (B) Representative photomicrographs show SA-Gal-positive cells (blue) in Td/TERTs (day I05) and Td/mocks (day 32). Original magnification $\times 100$. hTERT overexpression exceeded mitogenic potential. (C) Proliferative activity assay response to VEGF 165 . $(\mathbf{D})$ Proliferative activity assay response to bFGF.The increase in mitogenic response to angiogenic cytokines of Td/TERTs (day 73) was statistically significant compared with Td/mocks (day 59) (VEGF: $<$ 0.00I; bFGF: $<<0.001$ vs Td/mocks). 
A

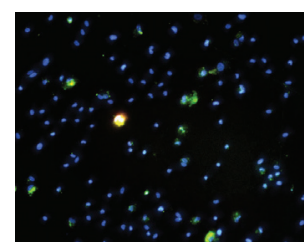

Td/TERT day 51

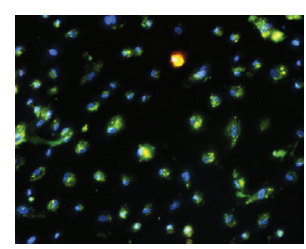

$\mathrm{Td} /$ mock

day 51

C

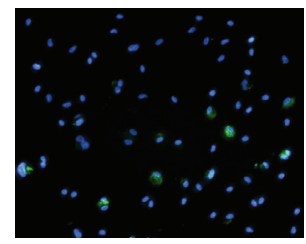

Td/TERT

day 59

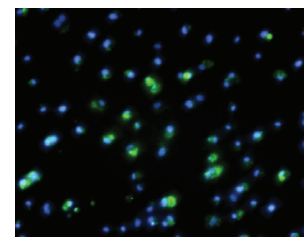

Td/mock

day 55
B

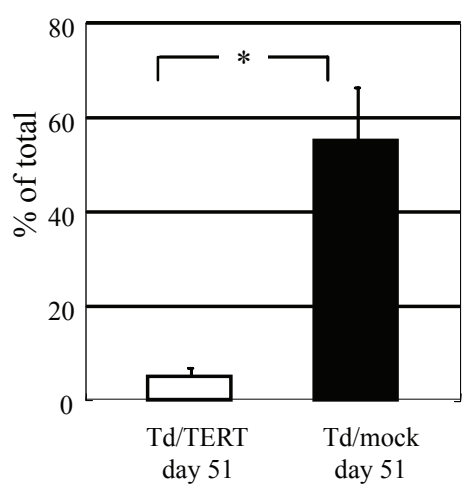

D

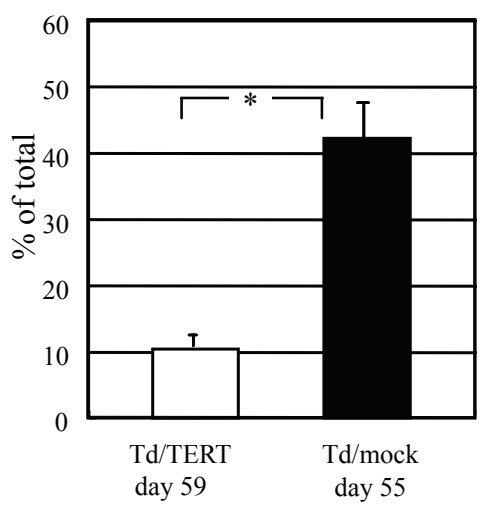

Figure 4 hTERT transduction contributes to cell survival. (A) Representative photomicrographs show Annexin-V (green), propidium iodide (red), and nuclei were stained with blue-fluorescent Hoechst 33342. Original magnification xI00. (B) Quantification of apoptosis-induced. The number of apoptotic cells was significantly increased in $\mathrm{Td} /$ mocks $\left(\mathrm{p}<0.00 \mathrm{I}\right.$ ) vs Td/TERTs. (C) Oxidative stress measurement in live cells. Representative photomicrographs show the cells stained with carboxy- $\mathrm{H}_{2} \mathrm{DCFDA}$ (green), corresponding to oxidized cells by ROS, and nuclei were stained with blue-fluorescent Hoechst 33342. Original magnification $x$ I00. (D) Quantification of oxidatively damaged cells. The oxidatively damaged cells significantly increased in Td/mocks $(p<0.00 I)$ vs Td/TERTs.

(at day 73) than that in Td/mocks (at day 59) (VEGF; 0.506 $\mathrm{nm} \pm 0.061 \mathrm{~nm}$ vs $0.239 \mathrm{~nm} \pm 0.013 \mathrm{~nm}, \mathrm{p}<0.001$, bFGF; $0.697 \mathrm{~nm} \pm 0.067 \mathrm{~nm}$ vs $0.261 \mathrm{~nm} \pm 0.027 \mathrm{~nm}, \mathrm{p}<0.001)$. These data suggest that Td/TERTs generate more remarkable angiogenic responses to angiogenic cytokines, perhaps as part of their general endothelial function, than that of $\mathrm{Td} /$ mocks.

\section{hTERT transduction contributes to cell survival}

To confirm whether hTERT modulates cell survival, cell apoptosis and oxidative damage were evaluated in transduced HUVECs. Strong Annexin-V (green) staining, corresponding to apoptotic cells, was observed in Td/mocks (Figure 4A). The staining indicated that serum starvation-induced apoptosis was markedly reduced in Td/TERTs (Figure 4, B) $(4.9 \% \pm 1.5 \%$ vs $54.9 \% \pm 11.0 \%, \mathrm{p}<0.001)$. Furthermore, strong carboxy- $\mathrm{H}_{2}$ DCFDA (green) staining, corresponding to oxidized by ROS, was observed in Td/mocks (Figure 4C). The oxidatively damaged cells significantly increased in $\mathrm{Td} /$ mocks $(10.3 \% \pm 2.2 \%$ vs $42.1 \% \pm 6.7 \%, \mathrm{p}<0.001)$ (Figure 4, D). Staining in blue indicated nuclei by Hoechst 33342 (Figure 4A, C) Taken together, these results suggest that hTERT overexpression in HUVECs contributes to resisting apoptosis and oxidative damage.

\section{Gene expression up-regulated in hTERT HUVEC}

Finally we examined gene levels of transduced HUVECs. To explore the genes involved in hTERT-induced cell survival, 
we applied cDNA microarray to transduced HUVECs. We compared the endothelial cell biology, angiogenesis, PI3K/ Akt signaling pathway, apoptosis, and cell cycle gene expression profiles of Td/mocks (day 46) and Td/TERTs (day 60). Microarray analysis revealed that hTERT overexpression altered expression of various genes related to apoptosis, cell survival, and signal transduction molecules. As shown in Table 2, survival signals such as PI3K/Akt related gene levels were enhanced in hTERT overexpressed HUVECs.

\section{Discussion}

Functional changes in senescent endothelial cells in vivo may play an important role in the pathophysiology of ageassociated vascular disorders. Thus, recovery of endothelial cell profiles could have possible clinical advantages in some cases. Recently, transduction of hTERT has been used to generate immortal cells. Various approaches have been used, none of which convert the cells to a transformed phenotype (Bodnar et al 1998; Jiang et al 1999; Morales et al 1999;

Table 2 Gene array analysis of transduced HUVECs

\begin{tabular}{|c|c|c|c|c|}
\hline Gene bank & Symbol & Gene name & Description & \\
\hline & & & AKT and PI3K Family Members and Their Regulators & \\
\hline NM02II58 & C20orf97 & TRB3 & Chromosome 20 open reading frame 97 & ++ \\
\hline NM002074 & GNBI & GNBI & Guanine nucleotide binding protein (G protein), beta polypeptide I & ++ \\
\hline NM000883 & IMPDHI & IMPDHI & IMP (inosine monophosphate) dehydrogenase I & +++ \\
\hline NM00554I & INPP5D & SHIP & Inositol polyphosphate-5- phosphatase, I 45kDa & ++ \\
\hline NM0I422I & MTCPI & MTCPI & Mature T-cell proliferation I & ++ \\
\hline NM006218 & PIK3CA & pl I0alpha & phosphoinositide-3kinase, catalytic, alpha polypeptide & ++ \\
\hline NM006219 & PIK $3 C B$ & pl IObeta & phosphoinositide-3kinase, catalytic, beta polypeptide & ++ \\
\hline NM003629 & PIK3R3 & p55 gamma & Phosphoinositide-3-kinase, regulatory subunit, polypeptide 3 ( $\mathrm{p} 55$, gamma) & +++ \\
\hline NMI8I897 & PPP2R3A & PPP2R3A & Protein phosphatase 2 (formerly $2 A$ ), regulatory subunit $B$, alpha & ++ \\
\hline NM006246 & PPP2R5E & PPP2R5E & Protein phosphatase 2, regulatory subunit B, (B56), epsilon isoform & ++ \\
\hline \multirow[t]{2}{*}{ NM002737 } & PRKCA & PKC alpha & Protein kinase C, alpha & +++ \\
\hline & & & PI3K-Dependent/AKT-Dependent Pathways & \\
\hline NM002093 & GSK3B & GSK3 & Glycogen synthase kinase 3 beta & ++ \\
\hline \multirow[t]{2}{*}{ NM005923 } & MAP3K5 & MEKK5/ASKI & Mitogen-activated protein kinase kinase kinase 5 & +++ \\
\hline & & & Myeloid/lymphoid or mixed-lineage leukemia (trithorax homolog, Drosophia); & \\
\hline NM005938 & MLLT7 & AFXI & translocated to,7 & ++ \\
\hline NM002467 & MYC & c-Myс & V-myc myelocytomatosis viral oncogene homolog (avian) & +++ \\
\hline NM003998 & NFKBI & $\mathrm{KBFI}$ & Nuclear factor of kappa light polypeptide gene enhancer in B-cells I (pI05) & +++ \\
\hline NM020529 & NFKBIA & IKBA/MAD-3 & Nuclear factor of kappa light polypeptide gene enhancer in B-cells inhibitor, alpha & +++ \\
\hline NM00I 278 & CHUK & IKKA/IKKI & Conserved helix-loop-helix ubiquitous kinase & +++ \\
\hline NM002576 & PAKI & Pakl & P2I/Cdc42/Racl-activated kinase I (STE20 homolog, yeast) & +++ \\
\hline \multirow[t]{2}{*}{ NM0 2296} & GAB2 & GAB2 & GRB2-associated binding protein 2 & +++ \\
\hline & & & Apoptosis Panel for Monitoring Cell Programmed Death and Survival & \\
\hline NM004536 & $\mathrm{BIRCI}$ & NAIP & Baculoviral IAP repeat-containing protein I (Neuronal apoptosis inhibitory protein) & ++ \\
\hline NM003810 & TNFSFIO & TRAIL & Tumor necrosis factor (ligand) superfamily member, 10 & + \\
\hline NM00370I & TNFSFII & TRANCE & Tumor necrosis factor (ligand) superfamily member, II & ++ \\
\hline NM0038II & TNFSF9 & 4-IBB-L & Tumor necrosis factor (ligand) superfamily member, 9 & + \\
\hline \multirow[t]{2}{*}{ NM00I56I } & TNFRSF9 & $4-I B B$ & Tumor necrosis factor receptor superfamily member, 9 & +++ \\
\hline & & & Stress \& Toxicity Panel for Monitoring the Type of Stress Induced & \\
\hline NM002983 & CCL3 & MIP-Ia/SCYA3 & Chemokine (C-C motif) ligane 3 (Macrophage inflammatory protein I-alpha) & + \\
\hline NM021027 & UGTIA9 & UGTIA9 & UDP glycosyltransferase I family, polypeptide A9 & ++ \\
\hline
\end{tabular}


Gagnon et al 2002; Yang et al 1999). Virus systems are the most efficient way to deliver genes to cells. However, the clinical applications of viral vectors are limited because of the intrinsic properties of viruses. Therefore, it seems that no-virus approaches of somatic gene delivery are preferable. In this regard, we tried to generate endothelial cell lines by ectopic expression of hTERT in HUVEC using a no-virus system.

Several interesting functional properties were noted in our experiments. First, Td/hTERT retained high level hTERT gene expression during long-term culture, and the cells stably expressed typical endothelial cell markers, including CD31, KDR, and eNOS. In agreement with other reports (Yang et al 1999; Nisato et al 2004; Freedman and Folkman 2004; Shao and Guo 2004), hTERT expression exhibited contact inhibition and serum-dependent cell proliferation, and did not show signs of tumorigenic transformation. Second, Td/hTERT cell lines had significant mitogenic activity in response to angiogenic cytokines compared with Td/mocks. Third, hTERT induced a delay in senescence and hTERT overexpressed cells appeared more resistant to stressed conditions. Fourth, a variety of genes related to cell survival were enhanced in $\mathrm{Td} /$ hTERT. In contrast to the immortalization shown in previous reports (Yang et al 1999; Nisato et al 2004; Freedman and Folkman 2004; Shao and Guo 2004), our system did not establish immortalized endothelial cells, but led to improved functional integrity as well as adenovirus transduction. For immortalization, Kiyono et al (1998) demonstrated that telomerase activity was not sufficient for immortalization of human kerationocyte or mammary epithelial cells. It likely that additional activities of antisenescent genes may be required to maintain functional integrity. Moreover, for gene transfer with potential therapeutic efficiency, immortalization is not necessarily valuable for clinical approaches.

Our results imply that telomerase inhibited functional alterations associated with senescence in endothelial cells. Thus we tried to study the molecular profile and mechanisms of apoptotic resistance and the ability to delay senescence.

Several factors, such as oxidative stress and DNA damage, have been shown to cause endothelial dysfunction. Recent studies showed that introduction of TERT into human vascular cells increased longevity and functional activity of endothelial cells (Yang et al 1999), suggesting that telomere function is necessary for endothelial function. Endothelial cell aging is linked to an increase in intracellular ROS formation, which in turn affects TERT localization and activity (Haendler et al 2004), and oxidative stress-caused perturbation of antioxidant mechanisms results in decreased telomeric integrity in endothelial cells (Kurz et al 2004). Moreover, reduction of nuclear TERT during endothelial cell aging increases the sensitivity of aged endothelial cells toward apoptotic stimuli (Assmus et al 2003). Thus recent studies have gradually elucidated the function of TERT in regulating a delay in endothelial cell senescence.

Our gene array data suggest that the PI3K/Akt-related gene levels were enhanced in Td/hTERT. The serine/threonine kinase Akt, activated by various growth and survival factors through a pathway that requires PI3K-dependent, has been shown to regulate multiple biological processes and also acts as an anti-apoptosis protein (Franke et al 1997; Crowder et al 1998; Eves et al 1998; Ulrich et al 1998). In case of endothelial cell, it has been shown to act downstream of angiogenic growth factors VEGF and angiopoietin, and contributes to endothelial cell survival, proper vessel development (Gerber et al 1998; Kontos et al 1998; Carmeliet et al 1999; Kim et al 2000; Papapetropoulos et al 2000), formation of vascular structures in vitro (Kureishi et al 2000), and regulates endothelial cell nitric oxide production in response to VEGF (Luo et al 2000). Recently, Zaccagnini et al (2005) demonstrated that telomerase mediates VEGF-dependent responsiveness in the rat ischemic hindlimb model. They introduced the idea that hTERT behaves as an angiogenic factor and a downstream effector of VEGF signaling, and that telomerase activity appears to be required for VEGFdependent remodeling of ischemic tissue at the capillary and arteriole level. They hypothesized that the function of hTERT, at least in vascular cells, might be controlled by a molecular network of autoregulatory loops in which growth factor production plays an important role.

Furthermore, recent studies suggest that ROS works as a regulator of signal transduction (Griendling and Ushio-Fukai 2000). Nishida et al $(2000,2002)$ reported that heterotrimeric $\mathrm{Gi} /$ o proteins are putative target molecules of ROS and the $\beta \gamma$-subunit of $\mathrm{G}$ protein ( $\mathrm{G} \beta \gamma$ ) liberated from $\mathrm{Gi}$ and Go by oxidative stress activates PI3K, which in turn leads to activation of Akt and ERK. They hypothesized that the intracellular signal transduction induced by the G $\beta \gamma$ of Gi and Go may protect cells against oxidative stress as part of their normal cellular function. It is possible that Td/hTERT obtained during normal cellular function against stress conditions, as well as stable nuclear TERT obtained during cell aging, might cause the activation of PI3K/Akt-related gene levels and protect cells against various stress conditions.

We are trying to determine the various potentials of hTERT in endothelial cells. The knowledge gained from the further study of telomerase overexpressed endothelial 
cells might help explain effects on cell survival. Further mechanistic investigations could contribute to improvements in vascular disorders in terms of the selection of cell sources when patients undergo cell transplantation.

\section{Acknowledgment}

We appreciate Dr. Hiromi Nishimura for providing the human umbilical cord samples, and Dr. Masakazu Ishikawa for technical support in part of RT-PCR primers.

\section{Disclosures}

None of the authors have any conflicts of interest to disclose.

\section{References}

Assmus B, Urbich C, Aicher A, et al. 2003. HMG-CoA reductase inhibitors reduce senescence and increase proliferation of endothelial progenitor cells via regulation of cell cycle regulatory genes. Cir. Res, 92:1049-55.

Bodnar AG, Ouellette M, Frolkis M, et al. 1998. Extension of life-span by introduction of telomerase into normal human cells. Science, 279:349-52.

Carmeliet P, Lampugnani MG, Moons L, et al. 1999. Targeted deficiency or cytosolic truncation of the VE-cadherin gene in mice impairs VEGFmediated endothelial survival and angiogenesis. Cell, 98:147-57.

Crowder RJ, Freeman RS. 1998. Phosphatidylinositol 3-kinase and Akt protein kinase are necessary and sufficient for the survival of nerve growth factor-dependent sympathetic neurons. J Neurosci, 18:2933-43.

Eves EM, Xiong W, Bellacosa A, et al. 1998. Akt, a target of phosphatidylinositol 3-kinase, inhibits apoptosis in a differentiating neuronal cell line. Mol Cell Biol, 18:2143-52.

Franke TF, Kaplan DR, Cantley LC, et al. 1997. Direct regulation of the Akt proto-oncogene product by phosphatidylinositol-3,4-bishosphate. Science, 275:665-8.

Freedman DA, Folkman J. 2004. Maintenance of G1 checkpoint controls in telomerase-immortalized endothelial cells. Cell Cycle, 3: 811-16.

Gagnon E, Cattaruzzi P, Griffith M, et al. 2002. Human vascular endothelial cells with extended life spans: in vitro cell response, protein expression, and angiogenesis. Angiogenesis, 5:21-33.

Gerber HP, McMurtrey A, Kowalski J, et al. 1998. Vascular endothelial growth factor regulates endothelial cell survival through the phosphatidylinositol 3'-kinase/Akt signal transduction pathway. Requirement for Flk-1/KDR activation. J Biol Chem, 273:30336-43.

Greider CW. 1990. Telomerase, telomerase and senescence. Bioessays, 12:363-9.

Griendling KK, Ushio-Fukai M. 2000. Reactive oxygen species as mediators of angiotensin II signaling. Regul Pept, 91:21-7.

Haendeler J, Hoffmann J, Diehl JF, et al. 2004. Antioxidants inhibit nuclear export of telomerase reverse transcriptase and delay replicative senescence of endothelial cells. Circ Res, 94:703-5.

Harley CB, Futcher AB, Greider CW. 1990. Telomerase shorten during ageing of human fibroblasts. Nature, 345:458-60.
Hastie ND, Dempster M, Dunlop MG. 1990. Telomere reduction in human colorectal carcinoma and with aging. Nature, 346:866-8.

Jiang XR, Jimenez G, Chang E, et al. 1999. Telomerase expression in human somatic cells does not induce changes associated with a transformed phenotype. Nat Genet, 21:111-14.

Kim I, Kim HG, So JN, et al. 2000. Angiopoietin-1 regulates endothelial cell survival through the phosphatidylinositol 3'-Kinase/Akt signal transduction pathway. Circ Res, 86:24-9.

Kiyono T, Foster SA, Koop JI, et al. 1998. Both Rb/p16INK4a inactivation and telomerase activity are required to immortalize human epithelial cells. Nature, 396:84-8.

Kontos CD, Stauffer TP, Yang WP, et al. 1998. Tyrosin 1101 of Tie2 is the major site of association of p85 and is required for activation of phosphatidylinositol 3-kinase and Akt. Mol Cell Biol, 18:4131-40.

Kureishi Y, Luo Z, Shiojima I, et al. 2000. The HMG-CoA reductase inhibitor simvastatin activates the protein kinase Akt and promotes angiogenesis in normocholesterolemic animaks. Nat Med, 6:1004-10.

Kurz DJ, Decary S, Hong Y, et al. 2004. Chronic oxidative stress compromises telomere integrity and accelerates the onset of senescence in human endothelial cells. J Cell Sci, 117:2417-26.

Luo Z, Fujio Y, Kureishi Y, et al. 2000. Acute modulation of endothelial Akt/PKB activity alters nitric oxide-dependent vasomotor activity in vivo. J Clin Invest, 106:493-9.

Morales CP, Holt SE, Ouellette M, et al. 1999. Absence of cancer-associated changes in human fibroblasts immortalized with telomerase. Nat Genet, 21:115-18.

Murasawa S, Llevadot J, Silver M, et al. 2002. Constitutive human telomerase reverse trascriptase expression enhances regenerative properties of expression enhances regenerative properties of endothelial progenitor cells. Circulation, 106:1133-9.

Nakamura TM, Morin GB, Chapman KB, et al. 1997. Telomerase catalytic subunit homologs from fission yeast and human. Science, 277: 955-9.

Nisato RE, Harrison JA, Buser R, et al. 2004. Generation and characterization of telomerase-transfected human lymphatic endothelial cells with an extended life span. Am J Pathol, 165:11-24.

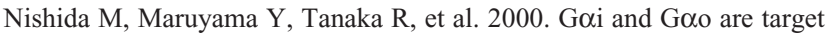
proteins of reactive oxygen species. Nature, 408:492-5.

Nishida M, Schey KL, Takagahara S, et al. 2002. Activation mechanism of Gi and Go by reactive oxygen species. J Biol Chem, 277:9036-42.

Papapetropoulos A, Fulton D, Mahboubi K, et al. 2000. Angiopoietin-1 inhibits endothelial cell apoptosis via the Akt/ survivin pathway. $J$ Biol Chem, 275:9102-5.

Shao R, Guo X. 2004. Human microvascular endothelial cells immortalized with human telomerase catalytic protein: a model for the study of invitro angiogenesis. Biochem Biophys Res Commun, 321:788-94.

Ulrich E, Duwel A, Kauffmann-Zeh A, et al. 1998. Specific TrkA survival signals interfere with different apoptotic pathways. Oncogene, $16: 825-32$.

Wright WE, Shay JW. 1992. The two-stage mechanism controlling cellular senescence and immortalization. Exp Gerontol, 27:383-9.

Yang J, Chang E, Cherry AM, et al. 1999. Human endothelial cell life extension by telomerase expression. J Biol Chem, 274:26141-8.

Zaccagnini G, Gaetano C, Della Pietra L, et al. 2005. Telomerase mediates vascular endothelial growth factor-dependent responsiveness in a rat model of hind limb ischemia. J Biol Chem, 280:14790-8. 